\title{
Synchronous Primary Carcinoma of the Colon - A case report and review of literature
}

\author{
Shekar Y tati a, Jagdish B ${ }^{\text {a }}$, Chidananda KV ${ }^{\text {a }}$, Harris Mohammed ${ }^{\mathrm{b}}$, \\ Akshay Pai ${ }^{\mathrm{b}}$, Somdeep Ghosh ${ }^{\mathrm{b}}$, Karthikeyan ${ }^{\mathrm{b}}$, Kariappa TM ${ }^{\mathrm{c}}$. \\ a, Professor, b, Post graduate, department of general surgery. c, Professor, department of pathology. KVG \\ medical college \& Hospital, Sullia - DK, Karnataka, India.
}

\begin{abstract}
Synchronous carcinoma of the colon is rare but not infrequent. The incidence ranges from 2-5\% . Synchronous primary carcinoma of the colon is defined as two or more primary colonic cancers are identified at the same time and confirmed by histological evaluation. The significance of synchronous primaries is not to miss second lesion, patients needs complete screening of the colon and excision of all the lesions. Patient may go for extended colectomy.

We came across one such case who presented with pain abdomen for one month and abdominal distension with vomiting for two days. On abdominal examination a hard lump was present in the right upper abdomen after upper gastro intestinal decompression was done by nasogastric tube aspiration.USG abdomen revealed thickening and narrowing of ascending colon and splenic flexure areas of the colon. Colonoscopy could not be negotiated beyond upper end of the descending colon. Exploratory laparotomy was done and two growths one at just above the caecum and another is just below the splenic flexure. Extended right hemicolectomy was done followed by end to end ileo-colostomy was done. Post operative period was uneventful.

We are presenting this case because of its rarity which demands complete evaluation of the colon by colonoscopy. if colonoscopy does not yield any result exploratory laporotomy and meticulous palpation of the colon is advised. We also reviewed the literature to freshen our knowledge regarding synchronous primary of colon.
\end{abstract}

Key words: Synchronous Primary Carcinoma of the Colon, Colonoscopy, Ultrasonogram (USG) abdomen and Extended right hemi colectomy.

\section{Introduction}

Synchronous primary carcinoma of the colon is rare but not infrequent. The incidence ranges from 2$5 \%$ of total colonic cancers [1-4]. Synchronous Primary is defined as more than one malignant lesion at the time of first diagnosis. Difficulties will be encountered in diagnosis and treatment. In one study $42 \%$ cases were diagnosed preoperatively, $24 \%$ were diagnosed during surgery and $34 \%$ diagnosed histologically from pathological specimen [2]. Difficult to arrive at a definite preoperative diagnosis because patient may present with acute or sub acute obstruction and demands semi emergency surgery. Palpation of entire colon is the best option during surgery to rule out synchronous primary. Patient may require extended colonic resection if synchronous primary is found and extended resection may lead to increased morbidity and mortality particularly in aged patients [14].

\section{Case Report}

A 35 year old male presented with pain abdomen for 2 months, abdominal distension and vomiting for 2 days. Patient gives $\mathrm{h} / \mathrm{o}$ significant weight loss with loss of appetite and loose stools.

On examination abdomen was distended, tender all over with increased bowel sounds. It was diagnosed as subacute obstruction. Patient was managed with NPO and i.v fluids, upper GIT decompression was done by NGT aspiration. Hard mass was palpable in the left hypochondrium once distension was subsided following conservative treatment. Rest of the abdomen was normal. Provisional diagnosis was carcinoma arising from splenic flexure resulting in subacute large bowel obstruction.

USG showed well defined hypoechoic bowel mass lesion in the splenic hilar region causing luminal narrowing. Similar lesion was found in the ascending colon with diffuse circumferential wall thickening of cecum and ascending colon. No free fluid and no organomegaly were noted. Impression was two synchronous primary malignant lesions. Colonoscopy was done; scope went up to upper part of the descending colon and not able to negotiate further because of luminal narrowing. Biopsy was taken from periphery of the narrowing, did not yield any positive result.

Patient was posted for explorative laparotomy. Bowel exploration revealed hard irregular mass of $7 \times 6$ $\mathrm{cm}$ in the ascending colon just above the caecum and another mass of $8 \times 7 \mathrm{~cm}$ in the splenic flexure. Pericolic 
and intermediate lymph nodes were enlarged. Liver free from secondaries, no peritonial seedling and no free fluid was present in the peritonial cavity.

An extended Rt hemicolectomy was done, including $15 \mathrm{~cm}$ of terminal ileum, caecum ascending, transverse colon including both hepatic and splenic flexures and $5 \mathrm{~cm}$ of descending colon (fig. 1). End to end ileo-colostomy (descending) was done and abdomen was closed in layers. Patient had uneventful recovery. Patient was followed for a month and advised for chemo and radiotherapy.

Gross specimen was showing two masses. The one is at ascending colon just above the caecum measuring $7 \times 5 \times 2 \mathrm{~cm}$ showing an ulceroproliferative growth. Cut section was showing gray white stenosing lesion (fig. 2) with areas of necrosis. Similar mass was located at splenic flexure, measuring $7 \times 6 \times 3 \mathrm{~cm}$.

Microscopic picture from both tumour masses showed features of malignant cell infiltration into the colonic mucosa. The malignant cells were arranged in sheets exhibiting glandular pattern. The tumour cells were reaching up to the serosa. Tumour deposits were noted in the lymph nodes. Impression: both lesions were showing moderately differentiated adenocarcinoma of the colon with secondary deposits in the lymph nodes.

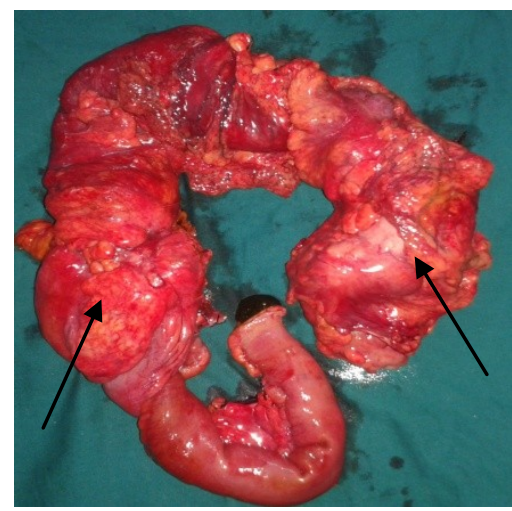

Figure 1 showing two growths : at caecum and the other at splenic flexure as the arrows are showing

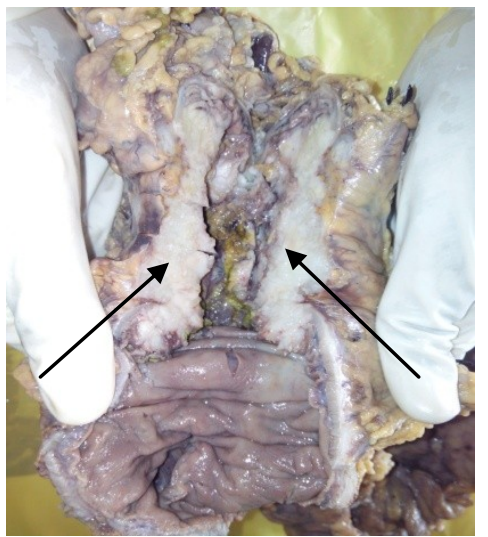

Figure 2 Cut section showing a grey white tubular lesion extending up to the serosa in the ascending colon

\section{Discussion}

Synchronous primary carcinoma is rare but not infrequent and it is well recognised clinical entity. The reported incidence in the literature accounts 2-5\% [1-4]. There is no consistent definition of synchronous colon cancer in the literature. In early studies synchronous and metachronous cancers were often mixed together as "multiple colon cancer" [2]. Some studies defined synchronous cancer as tumour arising six months after excision of primary tumour (Index tumour) the other defined it as the tumour arising one year after excision of primary tumour $[5,6]$. Strictly speaking presenting of two malignant lesions simultaneously in different part of the colon should be called as synchronous primary lesions. In our case one lesion was located in the ascending colon close to cecum and other lesion presenting just below the splenic flexure. Missed out malignant lesions during initial diagnosis and treatment of primary cancer will present as early synchronous carcinoma. Particularly impalpable mucosal lesions are likely to be missed out; therefore palpation of the colon during surgery, if possible per operative colonoscopy is necessary to diagnose such lesions.

Several studies revealed no significant difference in age distribution, gender incidence, clinical features and tumour behaviour between multiple and single colorectal cancers [4]. But some studies indicate synchronous colorectal cancers are more prevalent in males [7]. Synchronous colorectal cancers are also more 
common in older age group though younger age group are likely to get synchronous primary cancers (our patient is 35 years old) [2].

It is difficult to achieve accurate pre operative diagnosis in synchronous multiple colonic cancers. Most of the cases proximal lesion is likely to be missed. In one series only $42 \%$ of 59 synchronous cancer patients were diagnosed pre operatively, while $24 \%$ were detected per operatively, $34 \%$ were incidentally found on pathological specimen [8]. In another series only $66 \%$ synchronous cancer was diagnosed pre operatively [2]. In our case also we had diagnostic difficulty.

Colonoscopy is the best diagnostic procedure for identification of colonic malignant lesions, helps in confirming the diagnosis with high specificity and sensitivity [9]. However proximal growths are likely to be missed when distal growth is tubular and obstructing one where scope could not be negotiated further. In our case we had the same problem where scope was not able to pass further since growth was stenosing type. Other techniques like intra operative palpation, intra operative colonoscopy and barium enema have been evolved to assess the proximal colon. Though direct palpation is best method but mucosal and submucosal lesions are likely to be missed therefore most the surgeons prefer post surgery colonoscopy to evaluate complete colon [10].

Treatment part is also not having unified opinion. Subtotal or extended colectomy is the right option along with its mesocolon and lymph nodes [11]. Extended or subtotal colectomy will have increased morbidity. In our case also we did extended right hemicolectomy beyond splenic flexure along with mesocolon with intermediate lymph nodes. Some others believe in limited resection particularly in advanced age [12]. Rest of the chemo-radiation treatment is same as single cancer lesion.

Prognosis after surgery is also having variable opinions. Some claim no difference between single lesion and synchronous multiple lesion of the colonic cancer. However, survival rate in multiple synchronous growths is not worst [13] and others report survival rate is shorter in multiple synchronous cancers. However the prognosis with synchronous cases and that of single cases did not differ if the pathological stages were identical. [14].

\section{Conclusions}

Multiple synchronous lesions are not uncommon. Surgeons should be aware of the fact that per operative palpation of the entire colon is mandatory to diagnose the synchronous primary cancers. If doubt persists post operative colonoscopy is carried out to visualise the early or mucosal lesions of the entire colon. Morbidity is usually more since patients may go for extended colonic resection. The prognosis of synchronous cases and that of single cases did not differ if the pathological stages were identical.

\section{References}

[1]. Takeuchi H, Toda T, Nagasaki S, Kawano T, Minamisono Y, Maehara Y, Sugimachi K. Synchronous multiple colorectal adenocarcinoma. J Surg Oncol 1997; 64:304-7.

[2]. Chen HS, Sheen-Chen SM. Synchronous and "early" meta- chronous colorectal adenocarcinoma. Dis Colon Rectum 2000; 43:1093-9.

[3]. Wang HZ, Huang XF, Wang Y, Ji JF, Gu J. Clinical features, diagnosis, treatment and prognosis of multiple primary co- lorectal carcinoma. World J Gastroenterol 2004; 15:2136.

[4]. Papadopoulos V, Michalopoulos A, Basdanis G, et al. Synchronous and metachronous colorectal carcinoma. Tech Coloproctol 2004; 8:S97-100.

[5]. Kaibara N, Koga S, Jinnai D. Synchronous and metachronous malignancies of the colon and rectum in Japan with special reference to a coexisting early cancer. Cancer 1984;54: 1870 -

[6]. Oya M, Takahashi S, Okuyama T, Yamaguchi M, Ueda Y. Synchronous colorectal carcinoma: clinicopathological features and prognosis. Jpn J Clin Oncol 2003; 33:38-43.

[7]. Latournerie M, Jooste V, Cottet V, Lepage C, Faivre J, Bouvier AM. Epidemiology and prognosis of synchronous colorectal cancers. Br J Surg 2008; 95:1528-33.

[8]. Finan PJ, Ritchie JK, Hawley PR. Synchronous and 'early' metachronous carcinomas of the colon and rectum. Br J Surg 1987; 74:945-7.

[9]. Rex DK, Johnson DA, Lieberman DA, and Burt RW, Sonnenberg A. Colorectal cancer prevention 2000: screening recommendations of the American College of Gastroenterology. Am J Gastroenterol 2000; 95:868-77.

[10]. Evers BM, Mullins RJ, Matthews TH, Broghamer WL, Polk HC Jr. Multiple adenocarcinomas of the colon and rectum. An analysis of incidences and current trends. Dis Colon Rectum 1988;31:518-22.

[11]. Easson AM, Cotterchio M, Crosby JA, Sutherland H, Dale D, Aronson M, Holowaty E, Gallinger S. A population-based study of the extent of surgical resection of potentially curable colon cancer. Ann Surg Oncol 2002; 9:380-7.

[12]. Tsantilas D, Ntinas A, Petras P, Zambas N, Al Mogrambi S, Frangandreas G, Spyridis C, Gerasimidis T. Metachronous colorectal adenocarcinomas. Tech Coloproctol 2004; 8:s202-4.

[13]. Copeland EM, Jones RS, Miller LD. Multiple colon neoplasms. Prognostic and therapeutic implications. Arch Surg 1969; 98:141-3

[14]. Masatoshi Oya, Shuhei Takahashi, Takashi Okuyama, Masahiko Yamaguchi and Yoshihiko Ueda. Synchronous Colorectal Carcinoma: Clinico-pathological Features and Prognosis. Jpn J Clin Oncol 2003;33(1)38-43. 